\title{
Remarks on scale separation in flux vacua
}

\author{
F.F. Gautason, M. Schillo, T. Van Riet and M. Williams \\ Instituut voor Theoretische Fysica, KU Leuven, \\ Celestijnenlaan 200D, B-3001 Leuven, Belgium \\ E-mail: ffg@fys.kuleuven.be, marjorie@itf.fys.kuleuven.be, \\ thomas.vanriet@fys.kuleuven.be, m.williams@fys.kuleuven.be
}

ABSTRACT: We argue that the Maldacena-Nuñez no-go theorem excluding Minkowski and de Sitter vacua in flux compactifications can be extended to anti-de Sitter (AdS) vacua for which the Kaluza-Klein scale is parametrically smaller than the AdS length scale. In the absence of negative tension sources, scale-separated AdS vacua are ruled out in 11dimensional supergravity; in 10-dimensional supergravity, we show that such vacua can only arise in conjunction with large dilaton gradients. As a practical application of this observation we demonstrate that the mechanism to resolve O6 singularities in massive type IIA at the classical level is likely not to occur in AdS compactifications with scale separation. We furthermore remark that a compactification to four observable dimensions implies a large cosmological hierarchy.

KEYWORDS: Flux compactifications, D-branes

ARXiv EPrint: 1512.00457 


\section{Contents}

1 Introduction 1

2 What is required for separation of scales?

2.1 Scale separation in 11 dimensions 5

2.2 Scale separation in 10 dimensions 5

$\begin{array}{lll}2.3 & \text { Alternative perspective: integration with dilaton weights } & 7\end{array}$

3 An application: O6 singularities in massive type IIA $\quad 8$

4 Discussion $\quad 10$

\section{Introduction}

In any theory with extra dimensions, one is faced with the problem of stabilizing the extra dimensions at unobservable length scales. In string theory, fluxes provide a mechanism for stabilizing compact directions, however assuring that these compactifications are genuinely four-dimensional requires that the masses of the Kaluza-Klein (KK) modes are out of the reach of four-dimensional observers. It is believed that string theory provides a vast landscape of vacua which exhibit this separation of scales. ${ }^{1}$ This landscape, and a mechanism to populate it, is frequently employed to argue for an anthropic selection of the cosmological constant.

More concretely, a solution is scale separated if the KK-scale, $M_{\mathrm{KK}}$, can be decoupled from the scale of the cosmological constant, $M_{\Lambda}$ :

$$
\frac{M_{\Lambda}}{M_{\mathrm{KK}}} \ll 1
$$

For example, Minkowski vacua are automatically scale separated once the extra dimensions are compact, since $M_{\Lambda}=0$. However, in the most straightforward flux compactifications it is usually difficult (or impossible) to achieve this separation of scales.

There is a close connection between scale separation (1.1) and the cosmological constant problem, which is often stated as $M_{\Lambda} \ll M_{\text {Planck }}$. The appearance of the Planck scale in this inequality originates from the cut-off scale of a quantum field theory; usually the cut-off is taken to be the Planck mass since new physics must enter at that scale. However, if new physics enters before the Planck scale, it is more reasonable to state the cosmological constant problem in terms of this scale:

$$
\frac{M_{\Lambda}}{M_{\text {NewPhysics }}} \ll 1
$$

\footnotetext{
${ }^{1} \mathrm{~A}$ good overview can be found in $[1-3]$. Critical remarks about the mathematical evidence for a landscape can be found in [4].
} 
Clearly the KK-scale will introduce new physics in any theory with extra dimensions, therefore it is safe to treat $M_{\mathrm{KK}}$ as $M_{\text {NewPhysics. }}$ It therefore seems that the string landscape is by definition a landscape of vacua with a cosmological constant problem.

Loosely speaking, the flux vacua of the landscape can be divided into two classes: first, quantum corrected no-scale vacua and second, tree-level AdS vacua. The first class is based on the classical four-dimensional Minkowski solutions of [5, 6], which have moduli that are stabilized by perturbative and non-perturbative effects resulting in AdS vacua $[7,8]$, or perhaps even de Sitter vacua [9]. The second class of vacua have all moduli stabilised at the ten-dimensional supergravity level and have only been found in massive type IIA supergravity with intersecting O6 planes [10-14]). ${ }^{2}$

These two constructions have one property in common: some flux charges are cancelled by orientifold planes. One of the main points of this paper is to emphasize that this is no coincidence and that no scale separated vacua can be found in supergravity without the introduction of negative tension objects. The rough idea is as follows, the MaldacenaNuñez (MN) no-go theorem [17] (first pointed out in [18, 19]), shows that, at the level of ten-dimensional supergravity, flux compactifications can only lead to Minkowski or de Sitter vacua if negative tension objects (such as orientifolds) are present. Although the string landscape consists of many AdS vacua, they all exhibit a hierarchy of scales in order to be perceived as four-dimensional. Our expectation is that any mechanism that is required to get $\Lambda \geq 0$ is also at work for negative but small $\Lambda$. In other words: the same assumptions in the MN theorem that exclude Minkowski and de Sitter vacua can be used to exclude AdS vacua that are genuinely four-dimensional.

As an application of this idea we comment on a mechanism for resolving orientifold singularities found in [20,21]. The mechanism arose from a study of AdS flux vacua with scale separation within classical ten-dimensional supergravity. Using the findings in this note, we can argue that the classical resolution most likely does not occur when the AdS vacua feature scale separation.

\section{What is required for separation of scales?}

As discussed in the introduction, a realistic compactification of a theory with extra dimensions requires a hierarchy of scales. The mass of the lightest massive Kaluza-Klein mode must exceed that set by the cosmological constant, $M_{\mathrm{KK}} \gg M_{\Lambda}$. In order to verify this hierarchy for a given compactification, we must calculate both $M_{\mathrm{KK}}$ and the cosmological constant $\Lambda$. Although it is often easy to determine $\Lambda$, the calculation of $M_{\mathrm{KK}}$ requires detailed knowledge of the geometry of the internal manifold, $\mathcal{M}$. One must find the lowest-lying non-zero eigenvalue of the Laplacian on $\mathcal{M}$, but this is usually not feasible.

In order to set the stage, we write the $D$-dimensional metric as a warped product of a maximally symmetric 4 -dimensional metric and $d$ extra dimensions,

$$
d s^{2}=\mathrm{e}^{2 A(y)} \tilde{g}_{\mu \nu} d x^{\mu} d x^{\nu}+g_{m n} d y^{m} d y^{n} .
$$

\footnotetext{
${ }^{2}$ In type IIB similar constructions can be made but they are less understood $[15,16]$.
} 
The 4-dimensional metric is $g_{\mu \nu}=\mathrm{e}^{2 A(y)} \tilde{g}_{\mu \nu}$, where $\mu, \nu=0, \ldots, 3$, and $g_{m n}$ is the internal $d$-dimensional metric of the compact manifold $\mathcal{M}$, with $m, n=1, \ldots, d$. The full $D$ dimensional metric is denoted by $g_{M N}$ where $M, N=0, \ldots, D-1$. Since we take $\tilde{g}_{\mu \nu}$ to be maximally symmetric, the scale of the cosmological constant can be written simply in terms of the constant Ricci scalar of the unwarped metric. Specifically, the scale $M_{\Lambda}$ is defined via the observationally relevant vacuum energy density, $M_{\mathrm{Pl}}^{2} \Lambda$ :

$$
M_{\Lambda}^{4}=M_{\mathrm{Pl}}^{2} \Lambda=\frac{1}{4} M_{\mathrm{Pl}}^{2}\left|\tilde{R}_{4}\right|
$$

This unwarped curvature is related to the full 4-dimensional curvature via:

$$
\mathrm{e}^{4 A} R_{4}=\mathrm{e}^{2 A} \tilde{R}_{4}-\square_{d}\left(\mathrm{e}^{4 A}\right) .
$$

Furthermore, in any warped compactification, the observed value of the 4-dimensional Planck mass is:

$$
M_{\mathrm{Pl}}^{2}=M_{D}^{d+2} \int \sqrt{g_{d}} \mathrm{e}^{2 A} \mathrm{~d}^{d} y
$$

where $M_{D}$ is the $D$-dimensional Planck mass.

We estimate $M_{\mathrm{KK}}$ by the a warped internal curvature,

$$
M_{\mathrm{KK}}^{2} \sim \min \left[m_{\mathrm{KK}}^{2}(y)\right] \quad \text { where } m_{\mathrm{KK}}^{2}(y) \equiv e^{2 A} R_{d}
$$

where $R_{d}$ is the curvature scalar of the internal manifold. Our estimate of $M_{\mathrm{KK}}$ includes the effect of warping to account for gravitational redshifting within highly warped regions (in agreement with, e.g., [22]). Its dependence on the internal curvature is inspired by a theorem of Lichnerowicz [23] which states that a compact manifold with a bounded Ricci curvature

$$
R_{d} \geq(d-1) k>0,
$$

where $k>0$ is some constant, has a lower bound on the first non-zero eigenvalue of the Laplacian, ${ }^{3} \lambda_{1} \geq k d$. This is applicable to our case since we will see that the internal manifolds are positively curved. Our goal in this section is to find a bound on the ratio $M_{\mathrm{KK}} / M_{\Lambda}$ in a broad class of flux compactifications of 10- and 11-dimensional supergravity, thereby measuring separation of scales in these compactifications. The weak point in our argument will be the estimate above; our results will not hold for compactifications in which $M_{\mathrm{KK}}$ greatly differs from (2.5).

Now that $M_{\Lambda}$ and $M_{\mathrm{KK}}$ have been defined in terms of curvature scalars, we can use the Einstein equations to write them in terms of the matter content:

$$
\begin{aligned}
& R_{4}=\frac{d-2}{d+2} T_{4}-\frac{4}{d+2} T_{d} \\
& R_{d}=-\frac{d}{d+2} T_{4}+\frac{2}{d+2} T_{d}
\end{aligned}
$$

\footnotetext{
${ }^{3}$ The Lichnerowicz theorem applies to the Laplacian acting on scalar functions, in string theory one also encounters more general operators such as the Hodge-de Rham operator acting on $p$-form fields. Masses of the resulting modes will therefore be determined by the eigenvalues of the corresponding operators.
} 
where

$$
R_{4}=g^{\mu \nu} R_{\mu \nu}, \quad T_{4}=g^{\mu \nu} T_{\mu \nu}, \quad R_{d}=g^{m n} R_{m n}, \quad T_{d}=g^{m n} T_{m n} .
$$

In $[18,19]$ and later in $[17]$ it was realized that for theories with neither positive potentials nor negative tension sources, all terms on the right hand side of eq. (2.7) are negative definite, thus, ruling out de Sitter vauca. However, they did not rule out the possibility that $R_{4}$ is very small and negative, giving rise to a scale separated vacuum; this is exactly the possibility we are investigating.

The equations (2.7)-(2.8) play an important role in measuring scale separation, as we see below. Of course, the observationally relevant quantity is $M_{\Lambda}^{4}=M_{\mathrm{Pl}}^{2} \Lambda$, so we compare this to $M_{\mathrm{KK}}^{4}$ to obtain a dimensionless measure of scale separation. However, we find below that it is convenient to instead bound the right-hand side of:

$$
\frac{M_{\mathrm{KK}}^{4}}{M_{\mathrm{Pl}}^{2} \Lambda}<\frac{\left\langle m_{\mathrm{KK}}^{2}\right\rangle}{\Lambda},
$$

where

$$
\left\langle m_{\mathrm{KK}}^{2}\right\rangle=\frac{\int \sqrt{g_{d}} \mathrm{e}^{2 A} m_{\mathrm{KK}}^{2}(y) \mathrm{d}^{d} y}{\int \sqrt{g_{d}} \mathrm{e}^{2 A} \mathrm{~d}^{d} y} .
$$

The inequality follows from the fact that $M_{\mathrm{KK}}<M_{\mathrm{Pl}}$ for any well-posed KK analysis and $M_{\mathrm{KK}}^{2}<\left\langle m_{\mathrm{KK}}^{2}\right\rangle$. Therefore, the right-hand side of eq. (2.9) becomes

$$
\frac{\left\langle m_{\mathrm{KK}}^{2}\right\rangle}{\Lambda} \sim\left|\frac{\int \sqrt{g_{d}} \mathrm{e}^{4 A} R_{d} \mathrm{~d}^{d} y}{\int \sqrt{g_{d}} \mathrm{e}^{2 A} \tilde{R}_{4} \mathrm{~d}^{d} y}\right|=\left|\frac{\int \sqrt{-g} R_{d} \mathrm{~d}^{D} X}{\int \sqrt{-g} R_{4} \mathrm{~d}^{D} X}\right| \equiv\left|\frac{\int R_{d}}{\int R_{4}}\right| .
$$

We conclude that any scale-separated vacuum (i.e. a vacuum with $M_{\mathrm{KK}} \gg M_{\Lambda}$ ) must satisfy:

$$
\left|\frac{\int R_{d}}{\int R_{4}}\right| \gg 1
$$

If we are interested in AdS vacua possessing the same level of scale separation as our vacuum, we can estimate a lower bound of the left hand side of (2.9). A reasonable lower bound of the KK scale is $M_{\mathrm{KK}} \sim \mathrm{TeV}$ [24], and using the observed value of $\Lambda$, we see that the ratio is $10^{60}$.

Using equations (2.7)-(2.8) and other equations of motion we will show that in order to obtain this kind of hierarchy the same ingredients must be present as are required for a de Sitter or Minkowski compactification, i.e. orientifold planes or higher derivative corrections [25]. To do so we will work under almost the same assumptions as in [17]. First we specialize to 10- or 11-dimensional supergravity and exclude all higher-derivative corrections. Second, we assume that spacetime is a maximally symmetric four-dimensional manifold, while the remaining $d$-dimensional manifold is compact. Strictly speaking, [17] also applies to non-compact manifolds where the warp factor is allowed to go asymptotically to 0 , but we will not consider these cases.

We warm up by looking for scale separation in 11-dimensional supergravity, simply because it has fewer ingredients. After making the strategy clear in M-theory we will proceed to the 10-dimensional calculation where we also include the effect of D-branes. 
Our derivation is inspired by the derivation in [16], which applies to the special case of unwarped solutions in IIA/IIB with constant dilaton.

\subsection{Scale separation in 11 dimensions}

The bosonic part of the eleven-dimensional supergravity action is particularly simple:

$$
S_{11}=M_{11}^{9} \int d^{11} X \sqrt{-g}\left(R-\frac{1}{2}\left|F_{4}\right|^{2}\right)-\frac{1}{6} M_{11}^{9} \int F_{4} \wedge F_{4} \wedge A_{3},
$$

with

$$
\left|F_{n}\right|^{2}=\frac{1}{n !} F_{M_{1} M_{2} \cdots M_{n}} F^{M_{1} M_{2} \cdots M_{n}}, \quad F_{4}=\mathrm{d} A_{3} .
$$

Calculating the energy momentum tensor and plugging in eqs. (2.7) and (2.8) yields

$$
\begin{aligned}
& R_{4}=-\frac{4}{3}\left|F_{4}\right|^{2}-\frac{8}{3}\left|F_{7}\right|^{2}, \\
& R_{7}=\frac{5}{3}\left|F_{4}\right|^{2}+\frac{7}{3}\left|F_{7}\right|^{2} .
\end{aligned}
$$

In these expressions we only make use of magnetic fluxes, i.e. field strengths with legs entirely along the internal space. All external, or electric, fluxes that appear throughout the calculation are replaced by their magnetic duals, e.g. $F_{7}=\star_{11} F_{4}$.

We recognise that $R_{4} \leq 0$ as we expect from Maldacena-Nuñez and $R_{7} \geq 0$. Taking the integrated ratio we find:

$$
\left|\frac{\int R_{7}}{\int R_{4}}\right|=\frac{5 \int\left|F_{4}\right|^{2}+7 \int\left|F_{7}\right|^{2}}{4 \int\left|F_{4}\right|^{2}+8 \int\left|F_{7}\right|^{2}} \leq \frac{5}{4} .
$$

This obviously does not satisfy the requirement (2.12) and we conclude that scale separation is not possible in M-theory with only fluxes. Despite the result (2.16), many scale separated vacua with fluxes exist in M-theory, see for instance [26], but crucially rely on higher derivative corrections, which we ignore.

It is somewhat surprising that we find such a strong bound on the curvature scalars in M-theory, given that constructions in massless type IIA with O6 planes uplift to purely geometric compactifications with fluxes in 11 dimensions. Although it is counterintuitive, the no-gos of [17-19] can also be applied to such constructions, ruling out Minkowski and de Sitter compactifications. This is confirmed by the results of [27], where it was shown in 10 dimensions that O6 compactifications, with all fluxes turned on except the Romans mass, can never lead to de Sitter vacua or substantial amounts of inflation. The results of this section can be applied to these compactifications, ruling out scale separation even in the presence of orientifolds.

\subsection{Scale separation in 10 dimensions}

In this section we treat type II supergravity in the democratic formalism:

$$
S=M_{10}^{8} \int d^{10} X \sqrt{-g}\left(R-\frac{1}{2}(\partial \phi)^{2}-\frac{1}{2} \mathrm{e}^{-\phi}\left|H_{3}\right|^{2}-\frac{1}{4} \sum_{n} \mathrm{e}^{\frac{5-n}{2} \phi}\left|F_{n}\right|^{2}\right),
$$


where in the sum over $p$ we have

$$
n= \begin{cases}1,3,5,7,9 & \text { type IIB } \\ 0,2,4,6,8,10 & \text { type IIA }\end{cases}
$$

and $F_{0}$ is the Romans mass. In the democratic formulation the duality relation between RR field strengths must be imposed on-shell

$$
F_{10-n}=\mathrm{e}^{\frac{5-n}{2} \phi}(-1)^{\frac{(9-n)(8-n)}{2}} \star_{10} F_{n} .
$$

We use this relation to treat all fluxes as internal, or magnetic. This is not strictly necessary, however in all cases one can dualize the result so that only magnetic fluxes appear.

For completeness we will also include the effects of localized D-branes. This amounts to adding

$$
S_{\mathrm{loc}}=-N_{p} \mu_{p} \int_{\Sigma_{p+1}}\left\{\mathrm{e}^{\frac{p-3}{4} \phi} \sqrt{-P[g]}+(-1)^{\frac{p(p-1)}{2}} P\left[C_{p+1}\right]\right\}
$$

to the action (2.17), representing $N_{p}$ D-branes of charge $\mu_{p}$ coupled to the gauge potential $C_{p+1}$, given by:

$$
F_{p+2}=\mathrm{d} C_{p+1}-H_{3} \wedge C_{p-1} .
$$

The symbol $P[\cdots]$ denotes the pullback of the corresponding bulk fields onto the submanifold $\Sigma_{p+1}$ which the brane wraps. Note that in order to preserve Lorentz invariance, the brane must wrap the 4 spacetime dimensions and $p-3$ internal directions. Calculating the energy-momentum tensor of the matter fields and sources and plugging them into eqs. (2.7) and (2.8) we find:

$$
\begin{aligned}
& R_{6}=\frac{1}{2}(\partial \phi)^{2}+\frac{3}{4} \mathrm{e}^{-\phi}\left|H_{3}\right|^{2}+\sum_{n \leq 6} \frac{3+n}{8} \mathrm{e}^{\frac{5-n}{2} \phi}\left|F_{n}\right|^{2}+\sum_{p \geq 3} \frac{15-p}{8} N_{p} \mu_{p} \mathrm{e}^{\frac{p-3}{4} \phi} \delta\left(\Sigma_{p+1}\right), \\
& R_{4}=-\frac{1}{2} \mathrm{e}^{-\phi}\left|H_{3}\right|^{2}-\sum_{n \leq 6} \frac{n-1}{4} \mathrm{e}^{\frac{5-n}{2} \phi}\left|F_{n}\right|^{2}-\sum_{p \geq 3} \frac{7-p}{4} N_{p} \mu_{p} \mathrm{e}^{\frac{p-3}{4} \phi} \delta\left(\Sigma_{p+1}\right)
\end{aligned}
$$

where $\delta\left(\Sigma_{p+1}\right)$ is a Dirac delta function that localizes the D-brane contribution onto the brane worldvolume. Again, we see that $R_{6}>0$ and $R_{4}<0$ as expected from the no-go theorems of [17-19].

To simplify the following equations we define

$$
\begin{aligned}
h & =\int \mathrm{e}^{-\phi}\left|H_{3}\right|^{2}, & f_{n} & =\int \mathrm{e}^{\frac{5-n}{2} \phi}\left|F_{n}\right|^{2}, \\
d_{p} & =N_{p} \mu_{p} \int_{\Sigma_{p+1}} \mathrm{e}^{\frac{p-3}{4} \phi} \sqrt{-P[g]}, & \sigma & =\int(\partial \phi)^{2} .
\end{aligned}
$$

The integrated curvatures then reduce to

$$
\begin{aligned}
& \int R_{6}=\frac{1}{2} \sigma+\frac{3}{4} h+\sum_{n} \frac{3+n}{8} f_{n}+\sum_{p} \frac{15-p}{8} d_{p}, \\
& \int R_{4}=-\frac{1}{2} h-\sum_{n} \frac{n-1}{4} f_{n}-\sum_{p} \frac{7-p}{4} d_{p} .
\end{aligned}
$$


We will now measure scale separation, as before, by examining the ratio of the curvatures:

$$
\left|\frac{\int R_{6}}{\int R_{4}}\right|=\frac{\frac{1}{2} \sigma+\frac{3}{4} h+\sum_{n} \frac{3+n}{8} f_{n}+\sum_{p} \frac{15-p}{8} d_{p}}{\frac{1}{2} h+\sum_{n} \frac{n-1}{4} f_{n}+\sum_{p} \frac{7-p}{4} d_{p}} .
$$

This expression suggests a number of ways to construct a vacuum with a large hierarchy of scales: in type IIA one can seemingly arrange for the Romans mass (in this equation $f_{0}$ ) to cancel the fluxes so as to obtain a small (or even positive) cosmological constant. This point was specifically addressed in [17] where it was shown that the equations of motion forbid such a cancellation. A more promising avenue is to exploit the fact that contributions from the dilaton, $\sigma$, the type IIB axion, $f_{1}$, and D7-branes, $d_{7}$, only appear in the numerator of eq. (2.24). One can then dial up these contributions in order to obtain a large ratio. This suggests that type IIB/F-theory compactifications could have an abundance of scale separated vacua, provided they can be stabilized with fluxes.

Using the dilaton equation of motion, we can further simplify this expression. Going back to (2.17) and (2.19) we find the integrated dilaton equation of motion implies

$$
\frac{1}{2} h-\sum_{n} \frac{5-n}{4} f_{n}-\sum_{p} \frac{p-3}{4} d_{p}=0 .
$$

Using this to replace $h$ in $(2.24)$ we find:

$$
\left|\frac{\int R_{6}}{\int R_{4}}\right|=\frac{\frac{1}{2} \sigma+\sum_{n} \frac{9-n}{4} f_{n}+\sum_{p} \frac{3+p}{4} d_{p}}{\sum_{n} f_{n}+\sum_{p} d_{p}} .
$$

We now see that the only possibility for a hierarchy of scales is for

$$
\sigma \gg \sum_{n} f_{n}+\sum_{p} d_{p}
$$

Otherwise, if the dilaton gradients can be neglected, (2.26) is bounded by three. ${ }^{4}$

\subsection{Alternative perspective: integration with dilaton weights}

A complementary means reaching the conclusion above is to integrate all quantities with some non-trivial weighting. Rather than integrating the curvatures (and the related equations of motion) solely with respect to the metric volume form $\sqrt{-g}$, one can integrate with respect to $e^{a \phi} \sqrt{-g}$, where the constant $a$ is chosen such that the combination $e^{a \phi} \sqrt{-g} g^{M N}$ is invariant under the constant rescaling

$$
g_{M N} \rightarrow s g_{M N}, \quad e^{-\phi} \rightarrow s^{2} e^{-\phi} \quad F_{n} \rightarrow s^{\frac{3-n}{4}} F_{n} .
$$

This rescaling is a symmetry of the classical equations of motion in the absence of sources, and it is the combination $\sqrt{-g} g^{M N}$ which is relevant in determining how the integrated curvatures $\int R_{4}$ and $\int R_{6}$ scale under it. Invariance of the integrated curvatures dictates that $a=2$ is the appropriate choice.

\footnotetext{
${ }^{4}$ This conclusion can be reached in analogy with centre of mass computations; a weighted average, $r_{\mathrm{com}}=\sum r_{i} m_{i} / \sum m_{j}$, is bounded by the largest contribution, $r_{i}$.
} 
Introducing a superscript ' $(\phi)$ ' to denote the weighting, i.e.

$$
\begin{array}{ll}
h^{(\phi)}=\int \mathrm{e}^{\phi}\left|H_{3}\right|^{2}, & f_{n}^{(\phi)}=\int \mathrm{e}^{\frac{9-n}{2} \phi}\left|F_{n}\right|^{2}, \\
d_{p}^{(\phi)}=N_{p} \mu_{p} \int_{N_{p+1}} \mathrm{e}^{\frac{p+5}{4} \phi} \sqrt{-P[g]}, & \sigma^{(\phi)}=\int e^{2 \phi}(\partial \phi)^{2} .
\end{array}
$$

we find that the integrated Einstein and dilaton equations of motion become

$$
\begin{aligned}
\int e^{2 \phi} R_{6} & =\frac{1}{2} \sigma^{(\phi)}+\frac{3}{4} h^{(\phi)}+\sum_{n} \frac{3+n}{8} f_{n}^{(\phi)}+\sum_{p} \frac{15-p}{8} d_{p}^{(\phi)}, \\
\int e^{2 \phi} R_{4} & =-\frac{1}{2} h^{(\phi)}-\sum_{n} \frac{n-1}{4} f_{n}^{(\phi)}-\sum_{p} \frac{7-p}{4} d_{p}^{(\phi)}
\end{aligned}
$$

and

$$
-\sigma^{(\phi)}+\frac{1}{2} h^{(\phi)}-\sum_{n} \frac{5-n}{4} f_{n}^{(\phi)}-\sum_{p} \frac{p-3}{4} d_{p}^{(\phi)}=0
$$

respectively. (The $\sigma^{(\phi)}$ term in the latter arises from integrating the $\square \phi$ term by parts.) Eliminating $h^{(\phi)}$ using the dilaton equation as before, we find that the ratio of curvatures becomes

$$
\left|\frac{\int e^{2 \phi} R_{6}}{\int e^{2 \phi} R_{4}}\right|=\frac{2 \sigma^{(\phi)}+\sum_{n} \frac{9-n}{4} f_{n}^{(\phi)}+\sum_{p} \frac{3+p}{4} d_{p}^{(\phi)}}{\sigma^{(\phi)}+\sum_{n} f_{n}^{(\phi)}+\sum_{p} d_{p}^{(\phi)}} .
$$

Therefore, integrating with respect to a weight that is invariant under the constant rescaling (2.28) ensures that the resulting integrated curvature ratio is always bounded by three (thus precluding scale-separated solutions). Nevertheless, it remains true that, if the dilaton has some wildly-varying profile in the internal dimensions, the ratio obtained in (2.32) will cease to be an appropriate estimate of the degree of scale separation. It is in this sense that this alternative approach agrees with the one used in deriving eq. (2.27).

\section{An application: $\mathrm{O} 6$ singularities in massive type IIA}

In this section we demonstrate that our findings provide concrete insights for type IIA flux compactifications. It has been shown in [12] (see also [10, 11, 13, 14]), that O6 compactifications of massive type IIA supergravity can have the following striking features: first, full moduli stabilisation at the classical level and second, an AdS vacuum where a parametric separation of scales simultaneously suppresses derivative and loop corrections. The parameter that allows for tuning is the flux quantum number, $n$, of the RR 4-form flux, $F_{4}$, that is not constrained by any tadpole condition. In particular one finds the following dependence of the string coupling, KK scale and cosmological constant on $n$ :

$$
e^{\phi} \sim \frac{1}{n^{3 / 4}}, \quad \frac{M_{\mathrm{KK}}^{2}}{M_{\mathrm{Pl}}^{2}} \sim \frac{1}{n^{7 / 2}}, \quad \frac{M_{\Lambda}^{2}}{M_{\mathrm{Pl}}^{2}} \sim \frac{1}{n^{9 / 2}} .
$$

This good news comes at a cost: the backreaction of the O6-planes is only captured in the limit where they are smeared over the compact dimensions. The validity of this approximation has been questioned in [28]. Unlike the standard type IIB compactifications 


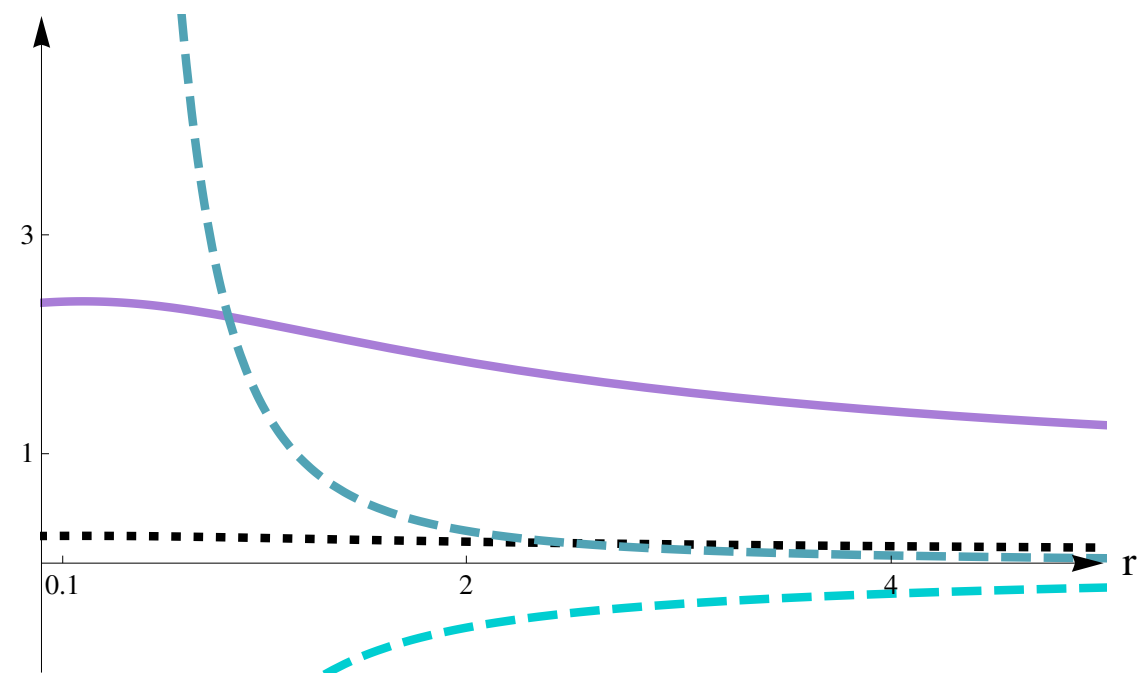

Figure 1. Figure 1b from [20]. The horizontal axis denotes the distance from the O6 source when the solution is locally approximated to be spherically symmetric. The dotted line corresponds to the string coupling and is practically flat. The solid line is the warpfactor and indeed stays finite, indicating a smooth geometry.

to 4-dimensional Minkowski space [5, 6], the backreaction has not been computed since the compactification involves four intersecting O6-planes. Even in flat space, supergravity solutions describing 4 mutually orthogonal localised branes/planes are not known.

In reference [20] it was demonstrated that the solutions [10-14] become full-blown dynamic SU(2) structures when the O6-planes are localised. Although the dynamic SU(2) structure implies that the solution is hopelessly complicated, reference [20] provides some reason for optimism: despite the presence of O6-planes, the fully back-reacted metric can be regular. This is achieved at the 10-dimensional level when the Roman's mass is non-zero, contrary to the usual massless resolution via an uplift to pure geometry in 11 dimensions.

We now argue that this resolution is unlikely to occur when back-reacting the O6planes of [12]. We do not claim that the resolution mechanism of [20] is invalid, ${ }^{5}$ instead, we claim it does not occur in vacuum solutions with scale separation. Our reasoning is rather simple. If the geometry is smooth in 10 dimensions, then the bulk field equations are satisfied without the need for explicit source terms. But without sources - particularly negative tension sources - our results indicate that scale separation requires large dilaton variation, a feature absent in the resolved O6 solutions of [20]. For clarity we have added figure 1 of [20] showing the profile of the dilaton. ${ }^{6}$ From the figure one concludes that the dilaton is nearly constant. The authors of [20] also solve the system perturbatively around the massless O6 solution and find that the dilaton is constant at first order.

\footnotetext{
${ }^{5}$ In fact some extra evidence for the existence of the resolution mechanism was found from a field theory viewpoint [21].

${ }^{6}$ We are grateful to the authors of [20] for allowing us to reproduce this figure.
} 


\section{Discussion}

The purpose of this note is to establish conditions for constructing genuinely lowerdimensional vacua. Our calculation applies to 10- and 11-dimensional supergravity with fluxes, excluding higher-derivative corrections. We find that in the absence of the negative tension induced by orientifold singularities, the level of scale separation is controlled by dilaton gradients in 10-dimensional supergravity. In 11-dimensional supergravity we show that scale separation is impossible. This shows that the usual no-go theorems of [17-19] against Minkowski and de Sitter vacua from fluxes extend to AdS vacua with small values of the cosmological constant compared to the KK scale. This provides a natural explanation for the observation in [29] that a class of warped AdS vacua has no solutions which exhibit scale separation. We also demonstrate that the classical resolution of O6 singularities due to Romans mass [20,21] cannot take place in a compactification with scale separation such as those of [12]. This is because these constructions do not exhibit the large dilaton gradients that provide the only hope for scale separation in constructions without negative tension sources. Finally, our results have immediate applications for model building.

As discussed in the introduction, any scale separated vacuum in a theory with extra dimensions will have a cosmological hierarchy problem. This statement has implications for the string landscape. In a theory with a landscape of vacua, many apparent fine-tuning problems are solved by the anthropic principle. To avoid any use of anthropic reasoning, either the landscape or a mechanism to populate it, e.g. eternal inflation, should be absent from the theory. As observers in a particular vacuum, the difficulty lies in identifying when to apply anthropic reasoning. It is widely accepted that the cosmological constant problem can be solved by anthropic reasoning [30], whereas the number of macroscopic spacetime dimensions might have a dynamical explanation [31]. Given the relation between the cosmological hierarchy and scale separation, these viewpoints are inconsistent. So either we have to accept that there is a dynamical mechanism for selecting 4 large spacetime dimensions which implies a cosmological hierarchy (but softens it significantly), or the reason we observe 4 dimensions is anthropic. The first possibility is clearly the more exciting option.

It is natural to think about scale separated AdS vacua from a holographic perspective. In particular the questions, "When is the cosmological constant small with respect to the KK scale?" and "Is there a landscape of such solutions?" should have a clear translation in the dual CFT language. Some general properties of CFTs dual to scale separated AdS vacua are known [32-34], e.g. they would exhibit a parametrically large gap in the spectrum of dimensions of conformal operators. Although studying scale separation from the holographic viewpoint would be of great interest, no explicit CFT example is known.

\section{Acknowledgments}

We are happy to acknowledge useful discussions with N. Bobev, M. Kleban, S. Sethi and A. Tomassielo. TVR furthermore likes to thank the organisers of the workshops "FineTuning, Anthropics and the String Landscape" (Madrid 2014) and the "Gordon Research Conference: String Theory \& Cosmology" (Hong Kong 2015) for providing the right environment for thinking about the matters discussed in this paper. This work is supported 
by the National Science Foundation of Belgium (FWO) grant G.0.E52.14N Odysseus and postdoctoral fellowship programmes, and the European Union's Horizon 2020 research and innovation programme under the Marie Skłodowska-Curie grant agreement No 656491. We acknowledge support from the European Science Foundation HoloGrav Network, the Belgian Federal Science Policy Office through the Inter-University Attraction Pole P7/37, and the COST Action MP1210 'The String Theory Universe'.

Open Access. This article is distributed under the terms of the Creative Commons Attribution License (CC-BY 4.0), which permits any use, distribution and reproduction in any medium, provided the original author(s) and source are credited.

\section{References}

[1] F. Denef, Les Houches lectures on constructing string vacua, in String theory and the real world: from particle physics to astrophysics. Proceedings, Summer School in Theoretical Physics, $87^{\text {th }}$ Session, Les Houches France July 2-27 2007, pg. 483 [arXiv:0803.1194] [INSPIRE].

[2] M. Graña, Flux compactifications in string theory: a comprehensive review, Phys. Rept. 423 (2006) 91 [hep-th/0509003] [INSPIRE].

[3] M.R. Douglas and S. Kachru, Flux compactification, Rev. Mod. Phys. 79 (2007) 733 [hep-th/0610102] [INSPIRE].

[4] T. Banks, The top $10^{500}$ reasons not to believe in the landscape, arXiv:1208.5715 [INSPIRE].

[5] K. Dasgupta, G. Rajesh and S. Sethi, M theory, orientifolds and G-flux, JHEP 08 (1999) 023 [hep-th/9908088] [INSPIRE].

[6] S.B. Giddings, S. Kachru and J. Polchinski, Hierarchies from fluxes in string compactifications, Phys. Rev. D 66 (2002) 106006 [hep-th/0105097] [INSPIRE].

[7] S. Kachru, R. Kallosh, A.D. Linde and S.P. Trivedi, De Sitter vacua in string theory, Phys. Rev. D 68 (2003) 046005 [hep-th/0301240] [INSPIRE].

[8] V. Balasubramanian, P. Berglund, J.P. Conlon and F. Quevedo, Systematics of moduli stabilisation in Calabi-Yau flux compactifications, JHEP 03 (2005) 007 [hep-th/0502058] [INSPIRE].

[9] A. Westphal, De Sitter string vacua from Kähler uplifting, JHEP 03 (2007) 102 [hep-th/0611332] [INSPIRE].

[10] K. Behrndt and M. Cvetič, General $N=1$ supersymmetric flux vacua of (massive) type IIA string theory, Phys. Rev. Lett. 95 (2005) 021601 [hep-th/0403049] [INSPIRE].

[11] J.-P. Derendinger, C. Kounnas, P.M. Petropoulos and F. Zwirner, Superpotentials in IIA compactifications with general fluxes, Nucl. Phys. B 715 (2005) 211 [hep-th/0411276] [inSPIRE].

[12] O. DeWolfe, A. Giryavets, S. Kachru and W. Taylor, Type IIA moduli stabilization, JHEP 07 (2005) 066 [hep-th/0505160] [INSPIRE].

[13] B.S. Acharya, F. Benini and R. Valandro, Fixing moduli in exact type IIA flux vacua, JHEP 02 (2007) 018 [hep-th/0607223] [INSPIRE].

[14] C. Caviezel, P. Koerber, S. Körs, D. Lüst, D. Tsimpis and M. Zagermann, The effective theory of type IIA AdS $S_{4}$ compactifications on nilmanifolds and cosets, Class. Quant. Grav. 26 (2009) 025014 [arXiv: 0806.3458] [INSPIRE]. 
[15] C. Caviezel, T. Wrase and M. Zagermann, Moduli stabilization and cosmology of type IIB on SU(2)-structure orientifolds, JHEP 04 (2010) 011 [arXiv:0912.3287] [INSPIRE].

[16] M. Petrini, G. Solard and T. Van Riet, AdS vacua with scale separation from IIB supergravity, JHEP 11 (2013) 010 [arXiv:1308.1265] [INSPIRE].

[17] J.M. Maldacena and C. Núnez, Supergravity description of field theories on curved manifolds and a no go theorem, Int. J. Mod. Phys. A 16 (2001) 822 [hep-th/0007018] [InSPIRE].

[18] G.W. Gibbons, Aspects of supergravity theories, in XV GIFT Seminar on Supersymmetry and Supergravity, Gerona Spain June 4-11 1984 [INSPIRE].

[19] B. de Wit, D.J. Smit and N.D. Hari Dass, Residual supersymmetry of compactified D $=10$ supergravity, Nucl. Phys. B 283 (1987) 165 [INSPIRE].

[20] F. Saracco and A. Tomasiello, Localized O6-plane solutions with Romans mass, JHEP 07 (2012) 077 [arXiv: 1201.5378] [INSPIRE].

[21] F. Saracco, A. Tomasiello and G. Torroba, Topological resolution of gauge theory singularities, Phys. Rev. D 88 (2013) 045018 [arXiv:1305.2929] [INSPIRE].

[22] C.P. Burgess et al., Warped supersymmetry breaking, JHEP 04 (2008) 053 [hep-th/0610255] [INSPIRE].

[23] A. Lichnerowicz, Geométrie des groupes des transformations (in French), Dunod, Paris France (1958).

[24] CMS collaboration, Searches for new physics using the $t \bar{t}$ invariant mass distribution in pp collisions at $\sqrt{s}=8 \mathrm{TeV}$, Phys. Rev. Lett. 111 (2013) 211804 [Erratum ibid. 112 (2014) 119903] [arXiv: 1309.2030] [INSPIRE].

[25] M.R. Douglas and R. Kallosh, Compactification on negatively curved manifolds, JHEP 06 (2010) 004 [arXiv: 1001.4008] [INSPIRE].

[26] K. Becker and M. Becker, M theory on eight manifolds, Nucl. Phys. B 477 (1996) 155 [hep-th/9605053] [INSPIRE].

[27] M.P. Hertzberg, S. Kachru, W. Taylor and M. Tegmark, Inflationary constraints on type IIA string theory, JHEP 12 (2007) 095 [arXiv:0711.2512] [INSPIRE].

[28] J. McOrist and S. Sethi, M-theory and type IIA flux compactifications, JHEP 12 (2012) 122 [arXiv: 1208.0261] [INSPIRE].

[29] D. Tsimpis, Supersymmetric AdS vacua and separation of scales, JHEP 08 (2012) 142 [arXiv: 1206.5900] [INSPIRE].

[30] S. Weinberg, The cosmological constant problem, Rev. Mod. Phys. 61 (1989) 1 [InSPIRE].

[31] R.H. Brandenberger and C. Vafa, Superstrings in the early universe, Nucl. Phys. B 316 (1989) 391 [INSPIRE].

[32] J. Polchinski and E. Silverstein, Dual purpose landscaping tools: small extra dimensions in AdS/CFT, in Strings, gauge fields, and the geometry behind: the legacy of Maximilian Kreuzer, A. Rebhan, L. Katzarkov, J. Knapp, R. Rashkov and E. Scheidegger eds., (2009), pg. 365 [arXiv:0908.0756].

[33] K. Papadodimas, AdS/CFT and the cosmological constant problem, arXiv:1106.3556 [INSPIRE].

[34] S. de Alwis, R.K. Gupta, F. Quevedo and R. Valandro, On KKLT/CFT and LVS/CFT dualities, JHEP 07 (2015) 036 [arXiv:1412.6999] [INSPIRE]. 